\title{
Mucormicosis rinocerebral: reporte de un caso y revisión de la literatura
}

\author{
Rhinocerebral mucormycosis: a case report and literature review
}

\author{
Angélica Julián Castrejón,* Pablo Andrés Crespo Reinoso
}

\section{RESUMEN}

La mucormicosis es una infección fúngica producida por el orden de los mucorales de la familia de los zigomicetos, afecta principalmente a individuos inmunocomprometidos, tiene una alta tasa de mortalidad y morbilidad, un tratamiento temprano de la patología de base, control de la infección y el desbridamiento quirúrgico son clave para un tratamiento exitoso. Se presenta el caso de una paciente de 17 años que muestra un cuadro de cetoacidosis diabética y mucormicosis rinocerebral; se describe el tratamiento médico quirúrgico que se llevó a cabo.

Palabras clave: Mucormicosis, rinocerebral, mucorales, cetoacidosis.

\section{INTRODUCCIÓN}

El primer caso de mucormicosis fue descrito en 1885 por Paltauf, quien empleó el término mycosis mucorina, la incidencia varía entre 0.43 y 1.7 casos por millón de habitantes, con cierta preferencia por el sexo masculino, representa entre 8.3 y $13 \%$ de todas las infecciones fúngicas diagnosticadas postmorten. ${ }^{1,2}$

\section{ABSTRACT}

Mucormycosis is a fungal infection produced by the order of mucorales of the zygomycete family, mainly affects immunocompromised individuals, has a high mortality and morbidity rate, early treatment of the underlying pathology and infection are key to a successful this treatment. We present the case of a 17-year-old female patient who presents with a picture of diabetic ketoacidosis and rhinocerebral mucormycosis, describing the surgical medical treatment that was carried out.

Keywords: Mucormycosis, rhinocerebral, mucorales, ketoacidosis.

El término mucormicosis describe el espectro de infecciones subagudas, agudas y crónicas causadas por hongos angiotrópicos del orden mucorales, cerca de la mitad son producidas por Rhizopus seguidas de Mucor, Absidia, Rizomucor respectivamente. Después del Aspergillus, son los siguientes patógenos más comunes en pacientes con neoplasias hematológicas y trasplantados. ${ }^{1-4}$

* CMF. Adscrita del Hospital Regional de Alta Especialidad de Ixtapaluca.

‡ Residente de tercer año de Cirugía Maxilofacial Universidad Nacional Autónoma de México.

Correspondencia:

Pablo Andrés Crespo Reinoso

E-mail: pablocrespood@gmail.com 
Los mucorales son organismos ubicuos, se encuentran con frecuencia en el suelo, verduras, alimentos en descomposición, excrementos de animales. ${ }^{5}$

La inoculación ocurre principalmente a través de la inhalación, contacto directo del organismo con superficies descubiertas de piel y mucosas, transmisión intravenosa (usuarios de drogas), así como la ingestión de esporas, además se ha reportado luego de una extracción dental con un desenlace fatal. ${ }^{3,6-8}$

Los mucorales tienen esporas lo suficientemente pequeñas (3-11 $\mu \mathrm{m})$ para poder alcanzar la porción más distal de los alveolos pulmonares. Las esporas más grandes $(>10 \mu \mathrm{m})$ pueden alojarse en los cornetes nasales. ${ }^{9}$

La enfermedad se observa casi exclusivamente en pacientes con compromiso sistémico como diabetes mellitus, leucemia, pacientes con trasplantes de órganos, síndrome de inmunodeficiencia adquirida, quemaduras graves y con medicamentos inmunosupresores. Entre 70 y $80 \%$ de los pacientes con mucormicosis padecen diabetes, en huéspedes inmunocompetentes la actividad fagocítica normal proporciona una barrera adecuada contra esta infección. ${ }^{5,10}$

La mucormicosis se puede clasificar en: rinocerebral, pulmonar; cutánea; gastrointestinal y diseminada con diferentes tasas de mortalidad. En el tipo rinocerebral, la espora inhalada infecta la cavidad nasal, los senos paranasales y la mucosa palatina, se expande a lo largo de los vasos y los nervios, pudiendo alcanzar la órbita, fosa pterigopalatina, fosa craneal media y el seno cavernoso logrando llegar al cerebro (Tabla 1). ${ }^{6}$

Las cepas de los mucorales tienen gran afinidad hacia las arterias y se adhieren a la pared arterial, crecen a lo largo de la lámina elástica interna de los vasos sanguíneos causando trombosis, isquemia y necrosis de los tejidos circundantes, esto se produce mediante el reconocimiento específico de la proteína reguladora de glucosa 78 (PRG78) del receptor del huésped. El ligando fúngico que se une durante la invasión del endotelio pertenece a la familia de proteínas de revestimiento de esporas. Este reconocimiento causa la muerte celular del huésped por inducción de la endocitosis del hongo mediada por células endoteliales. ${ }^{5,7,11,12}$

En pacientes en estado de cetoacidosis, la hiperglucemia induce la glucosilación excesiva de proteínas como la transferrina y la ferritina, lo que disminuye su afinidad por el hierro, además hay una acumulación de cuerpos cetónicos como $\beta$-hidroxibutirato, el bajo $\mathrm{pH}$ en los vasos sanguíneos deteriora fuertemente la capacidad de la transferrina para quelar el hierro. La glucosa, el hierro y el $\beta$-hidroxibutirato propician el crecimiento del hongo, también inducen la expresión de GRP78 y de proteínas de revestimiento de esporas. ${ }^{7}$

Las zonas necróticas en la cavidad nasal, el paladar o en la cara son signos iniciales tan sólo en $50 \%$ de los pacientes. El diagnóstico definitivo requiere evidencia histopatológica de invasión fúngica. Se pueden usar varias técnicas como el tratamiento con hidróxido de potasio al $20 \%$, tinción con metenamina plata de Grocott, tinción con hematoxilina y eosina, tinción con ácido peryódico-Schiff para la detección microscópica directa de mucorales. ${ }^{5,9}$

\section{CASO CLÍNICO}

Paciente femenino de 17 años de edad que refiere iniciar padecimiento actual 60 días previos al ingreso a nuestra unidad hospitalaria con polidipsia, polifagia, poliuria y pérdida de peso involuntaria de 15 kilogramos. A los 45 días posteriores muestra aumento de volumen en región geniana derecha, la cual es tratada por odontólogo general con antibioticoterapia sin resolver, a los tres días siguientes presenta úlcera necrótica en paladar del lado derecho, decide acudir con cirujano maxilofacial de manera particular, quien realiza biopsia incisional de la úlcera palatina, obteniendo resultado histológico de mucormicosis, por lo que decide referirla a Urgencias del Hospital Regional de Alta Especialidad de Ixtapaluca, cursando con un cuadro de cetoacidosis diabética

Tabla 1: Prevalencia, mortalidad y diagnóstico tardío de las diferentes clases de mucormicocis.

\begin{tabular}{lccc}
\hline Clase & Prevalencia (\%) & Mortalidad (\%) & Diagnóstico en autopsia (\%) \\
\hline Rinocerebral & $44-49$ & $35-65$ & 9 \\
Pulmonar & $10-16$ & $11-68$ & 69 \\
Cutánea & $10-11$ & 16 & 0 \\
Gastrointestinal & $2-11$ & 100 & 100 \\
Diseminado & $6-11$ & 100 & 100 \\
\hline
\end{tabular}




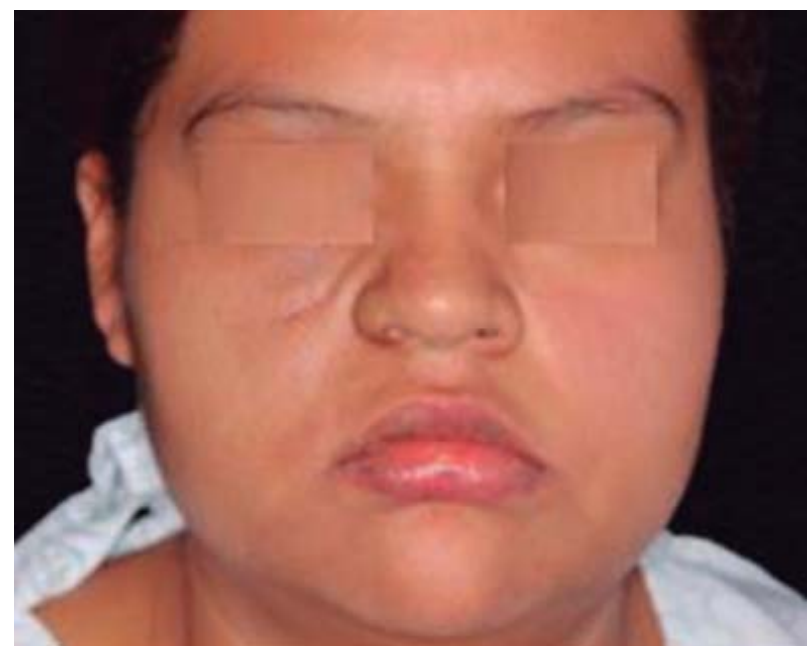

Figura 1: Fotografía facial inicial.

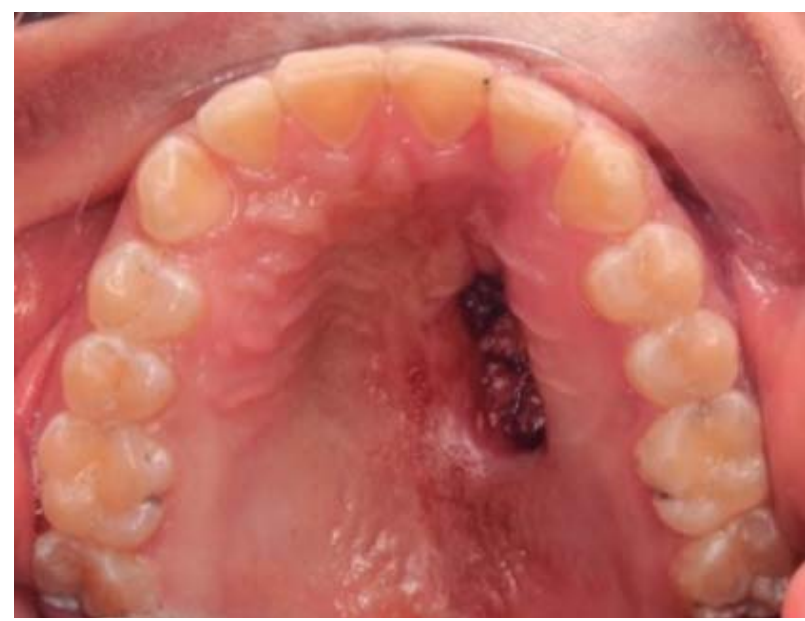

Figura 2: Úlcera palatina.

como hallazgo de relevancia, ya que desconocía su enfermedad. A la exploración física encontramos paciente de edad aparente mayor a la cronológica, distopia, lagoftalmos, epífora, movimientos oculares conservados, función muscular facial grado III derecha según escala House Brackmann, asimetría facial a expensas de depresión en región infraorbitaria derecha, parestesia del ala nasal, párpado inferior y hemilabio superior derecho con fístula infraorbitaria con ligera secreción serosa, mucosas deshidratadas, úlcera de aproximadamente 2 centímetros de longitud en paladar duro en región paramedial derecha (Figuras 1 y 2).

Una vez estabilizado el cuadro de cetoacidosis se inicia la terapia farmacológica a base anfotericina $B$ liposomal $60 \mathrm{mg}$ intravenoso cada 24 horas, insulina NPH 30 unidades por la mañana y 15 unidades por la noche, se realiza glucometría cada seis horas utilizando esquema de insulina regular de rescate:140-180 2UI; 181-220 4UI.

El tratamiento quirúrgico se lleva a cabo bajo anestesia general balanceada mediante abordaje

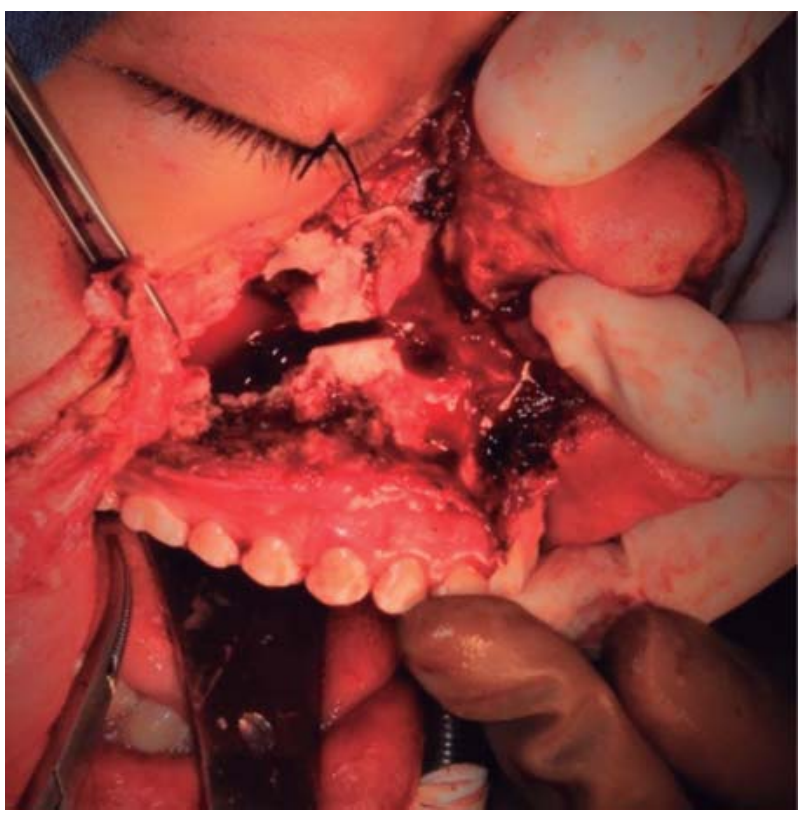

Figura 3: Maxilectomía.

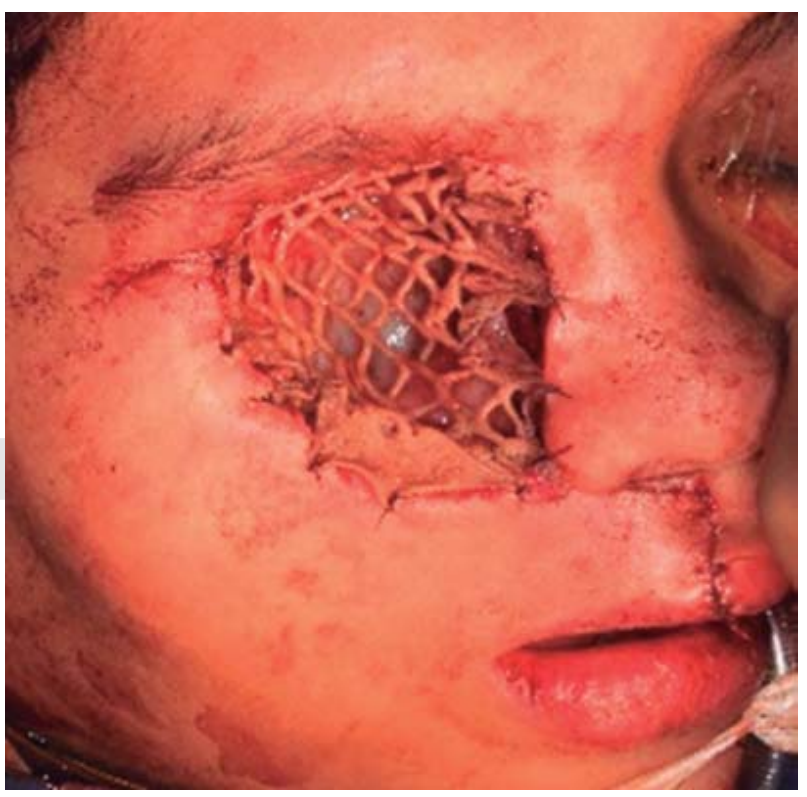

Figura 4: Injerto de piel. 
tipo Weber Ferguson, se realiza desbridamiento del tejido necrótico y escisión de tejido cutáneo en región geniana y ala de nariz del lado derecho, se envía a estudio histopatológico transoperatorio, el cual refiere área de inflamación aguda abscedada. A la búsqueda intencionada en corte por congelación y bajo observación de microscopio se identifican múltiples hifas, se procede a efectuar la hemimaxilectomía de infraestructura derecha mediante cierre reciprocante, se continúa con la escisión de piso orbitario, lo que favorece observar la grasa orbitaria, la cual presenta contenido seropurulento, se realiza exenteración orbitaria y eliminación de párpado superior e inferior, reposición de colgajos, rotación de pelotón adiposo bucal para conformación de piso nasal, colocación de injerto de piel en lecho quirúrgico orbitario (Figuras $3 y$ 4). Por último, se coloca obturador palatino quirúrgico por el equipo de prótesis maxilofacial, el cual tiene el objetivo principal de dividir la comunicación oronasal posterior a la maxilectomía de infraestructura, además de mantener estable el empaquetamiento quirúrgico con fines hemostáticos y permitir la alimentación vía oral, lo que evita la colocación de sondas para alimentación. Este tipo de obturador permanece cinco días y posterior al desempaquetamiento se coloca obturador transicional, mismo que se ajusta y será reemplazado por el obturador definitivo una vez alcanzada la cicatrización completa del defecto quirúrgico.

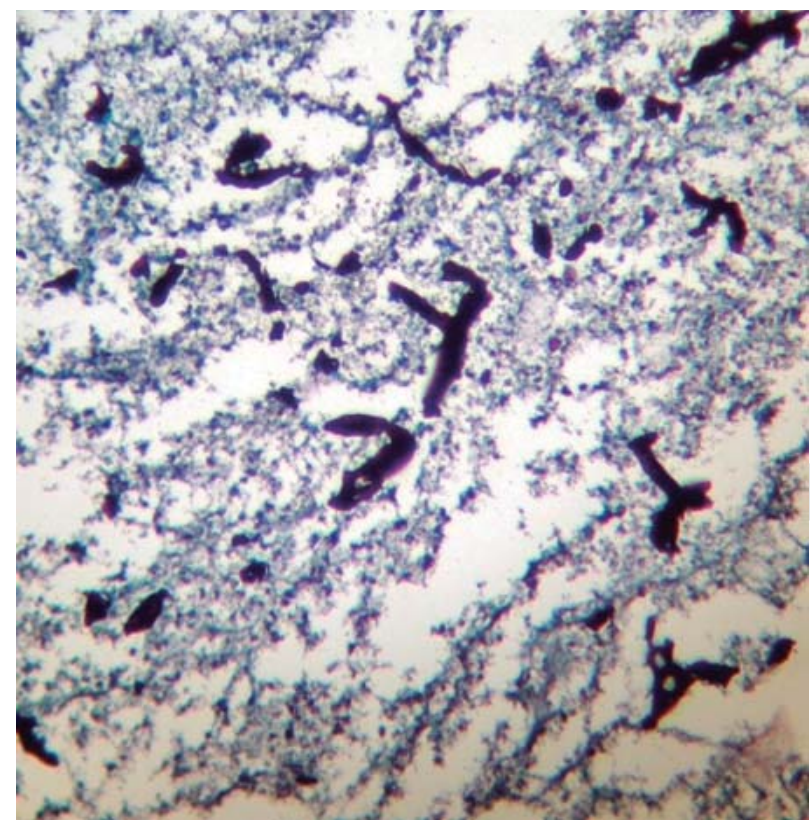

Figura 5: Tinción argéntica de Grocott.

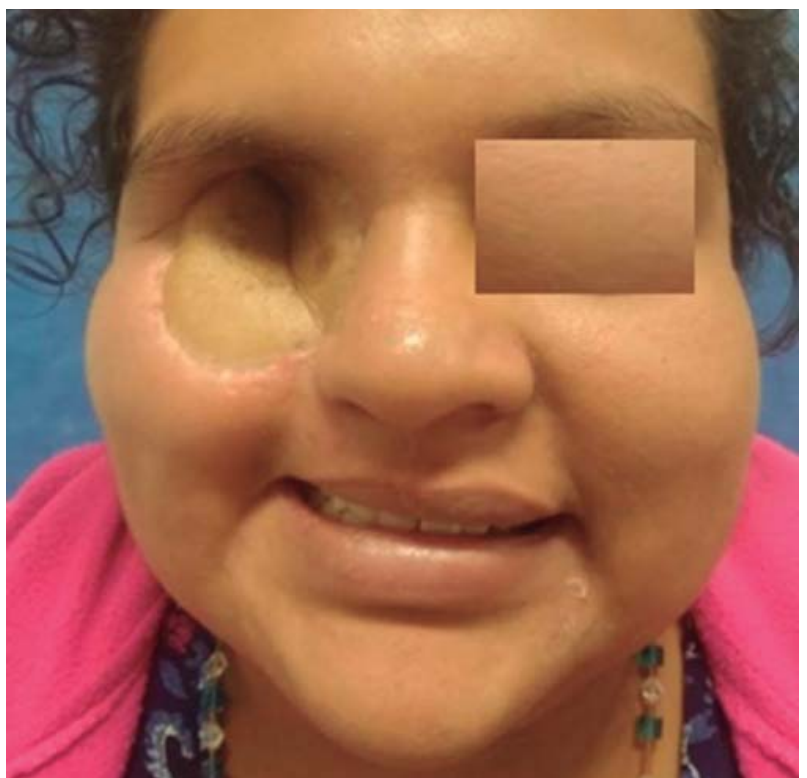

Figura 6: Postoperatorio a un año.

Se mantiene régimen de anfotericina durante 15 días postquirúrgicos. El estudio histopatológico final confirma el diagnóstico de mucormicosis con bordes libres de infiltración (Figura 5). En controles subsecuentes se observa adecuada evolución con buen proceso de cicatrización, (Figura 6) por lo que se remite al servicio de prótesis maxilofacial para continuar con tratamiento reconstructivo.

\section{DISCUSIÓN}

Entre los factores que influyen en el pronóstico está el diagnóstico oportuno en los primeros días, en nuestra paciente tuvimos un periodo de dos semanas sin un tratamiento antifúngico ni control para su enfermedad de base. Las características clínicas comunes son: fiebre, dolor de cabeza, dolor facial, secreción nasal, obstrucción nasal, úlceras, parálisis de nervios craneales, erosión y destrucción ósea. Los hallazgos en tomografía computarizada se observan típicamente como hipertrofia de los cornetes, la perforación de septum nasal y sinusitis en nuestra paciente coinciden con los signos y síntomas típicos de esta enfermedad, aunque no se reportó fiebre, presentó lagoftalmos y epífora. . $^{510}$

El desbridamiento quirúrgico del tejido infectado es crucial y debe realizarse con urgencia para limitar la propagación de la infección, la exenteración orbitaria siempre es un dilema para el cirujano, la evidencia actual no da indicaciones específicas; sin embargo, 
ante signos de invasión orbitaria como en el caso de nuestra paciente es preferible efectuarla. ${ }^{4,10,11}$

La aplicación intravenosa de anfotericina B es el antifúngico de elección en el tratamiento de la mucormicosis, la forma liposomal disminuye la toxicidad renal, además tiene una mejor penetración en los tejidos; sin embrago, no mejora el pronóstico de la enfermad, pero permite administrar por un periodo más largo con un perfil de efectos secundarios favorable cuando se compara con la anfotericina B convencional. Dosis mayores de $7.5 \mathrm{mg} / \mathrm{kg}$ no confieren mayor beneficio al paciente. El uso de posaconazol como terapia complementaria sigue siendo limitado, no se puede evaluar completamente su papel terapéutico debido al reducido número de informes que existen en la literatura. El tratamiento antifúngico y quirúrgico agresivo combinado se asoció con una disminución de la tasa de mortalidad a los 90 días, la terapia con anfotericina tardía incrementó la mortalidad hasta $83 \%$.,10,11

El oxígeno hiperbárico en estudios in vitro demostró inhibición del crecimiento fúngico y mayor actividad fungicida de anfotericina $B$, además de promover la liberación de factores de crecimiento, mejorar la angiogénesis y la cicatrización de heridas. ${ }^{4,9}$

El estudio histopatológico es fundamental para el diagnóstico de esta infección, la identificación a nivel de género especie es de importancia epidemiológica, pero no de relevancia. ${ }^{4,9,13,14}$

Los factores para un mal pronóstico incluyen afección intracraneal, hemiplejía, hemiparesia, afección de senos paranasales bilateral, úlceras palatina, necrosis facial e invasión orbitaria. ${ }^{4,14,15}$

\section{CONCLUSIONES}

La mucormicosis es una infección micótica rara con una alta tasa de morbilidad y mortalidad, el diagnóstico temprano es de suma importancia para el pronóstico de esta enfermedad. El tratamiento debe ser médico y quirúrgico, primero estabilizando la enfermedad de base; la administración de anfotericina $\mathrm{B}$ liposomal, la resección quirúrgica y el control de los factores de riesgo pueden mejorar el pronóstico. Siempre es necesario un enfoque multidisciplinario.

\section{BIBLIOGRAFÍA}

1. Metzen D, Böhm H, Zimmermann M, Reuther T, Kübler AC, Müller-Richter, UDA. Mucormycosis of the head and neck. J Craniomaxillofac Surg. 2012; 40 (8): e321-e327.

2. Petrikkos G, Skiada A, Lortholary O, Roilides E, Walsh TJ, Kontoyiannis DP. Epidemiology and clinical manifestations of mucormycosis. Clin Infect Dis. 2012; 54 (Suppl 1): S23-S34.

3. Camara-Lemarroy CR, González-Moreno El, Rodríguez-Gutiérrez R, Rendón-Ramírez EJ, Ayala-Cortés AS, Fraga-Hernández ML, Galarza-Delgado DA. Clinical features and outcome of mucormycosis. Interdiscip Perspect Infect Dis. 2014; 2014: 562610.

4. Jeong W, Keighley C, Wolfe R, Lee WL, Slavin MA, Kong DC, et al. The epidemiology and clinical manifestations of mucormycosis: a systematic review and meta-analysis of case reports. Clinical Microbiology and Infection. 2018, 25 (1): 26-34.

5. Swain SK, Sahu MC, Banerjee A. Non-sinonasal isolated facioorbital mucormycosis. A case report. J Mycol Med. 2018; 28 (3): 538-541.

6. Kudo K, Hasegawa H, Sato E, Kaneko T, Ishida D, Kanno C, Endo M. A case of rhinocerebral mucormycosis extending into the skull. Journal of Oral and Maxillofacial Surgery, Medicine, and Pathology. 2017; 29(1): 61-64.

7. Baldin C, Ibrahim AS. Molecular mechanisms of mucormycosis: the bitter and the sweet. PLoS Pathog. 2017; 13 (8). e1006408.

8. Acosta BC, Lucio LE, Uribe CA, Gómez MB. Mucormicosis rinocerebral de origen dental, reporte de un caso clínico y revisión de la literatura. Rev Esp Cirug Oral y Maxilofac. 2014; 36 (2): 6872.

9. Farmakiotis D, Kontoyiannis DP. Mucormycoses. Infect Dis Clin North Am. 2016; 30 (1): 143-163.

10. Therakathu J, Prabhu S, Irodi A, Sudhakar SV, Yadav VK, Rupa V. Imaging features of rhinocerebral mucormycosis: A study of 43 patients. The Egyptian Journal of Radiology and Nuclear Medicine. 2018; 49 (2): 447-452.

11. Elzein F, Kalam K, Mohammed N, Elzein A, Alotaibi FZ, Khan M, Albadani A. Treatment of cerebral mucormycosis with drug therapy alone: a case report. Med Mycol Case Rep. 2019; 23: 4-7.

12. Gebremariam T, Liu M, Luo G, Bruno V, Phan QT, Waring AJ et al. CotH3 mediates fungal invasion of host cells during mucormycosis. J Clin Invest. 2014; 124 (1): 237-250.

13. Kim J, Forston JK, Cook HE. A fatal outcome from rhinocerebral mucormycosis after dental extractions: a case report. J Oral Maxillofac Surg. 2001; 59: 693-697.

14. Pandey A, Bansal V, Asthana AK, Trivedi V, Madan M, Das A. Maxillary osteomyelitis by mucormycosis: report of four cases. Int $\mathrm{J}$ Infect Dis. 2011; 15 (1): e66-e69.

15. Malik AN, Bi WL, McCray B, Abedalthagafi M, Vaitkevicius $H$, Dunn IF. Isolated cerebral mucormycosis of the basal ganglia. Clin Neurol Neurosurg. 2014; 124: 102-105. 